Bull. Korean Math. Soc. 46 (2009), No. 3, pp. 439-446

DOI 10.4134/BKMS.2009.46.3.439

\title{
UNIVALENCE PROPERTIES FOR \\ A GENERAL INTEGRAL OPERATOR
}

\author{
DANIEL BReaZ
}

AbStRaCt. We consider the univalence function classes $\mathcal{T}, \mathcal{T}_{2}, \mathcal{T}_{2, \mu}$, and $\mathcal{S}(p)$. For these classes we shall study some univalence properties for a general integral operator. Furthermore we shall extend some known univalence criteria, i.e., Becker-type criteria.

\section{Introduction}

Let $\mathcal{U}=\{z \in \mathbb{C},|z|<1\}$ be the unit disk and $\mathcal{A}$ denotes the class of the functions $f$ of the form

$$
f(z)=z+a_{2} z^{2}+a_{3} z^{3}+\cdots, z \in \mathcal{U},
$$

which are analytic in the open disk, $\mathcal{U}$ and satisfy the condition $f(0)=f^{\prime}(0)-$ $1=0$. Consider $\mathcal{S}=\{f \in \mathcal{A}: f$ is univalent functions in $\mathcal{U}\}$.

Let $\mathcal{A}_{2}$ be the subclass of $\mathcal{A}$ consisting of functions is of the form

$$
f(z)=z+\sum_{k=3}^{\infty} a_{k} z^{k}
$$

Let $\mathcal{T}$ be the univalent subclass of $\mathcal{A}$ which satisfies

$$
\left|\frac{z^{2} f^{\prime}(z)}{(f(z))^{2}}-1\right|<1 \quad(z \in \mathcal{U}) .
$$

Let $\mathcal{T}_{2}$ be the subclass of $\mathcal{T}$ for which $f^{\prime \prime}(0)=0$. Let $\mathcal{T}_{2, \mu}$ be the subclass of $\mathcal{T}_{2}$ consisting of functions is of the form (1.1) which satisfy

$$
\left|\frac{z^{2} f^{\prime}(z)}{(f(z))^{2}}-1\right| \leq \mu \quad(z \in \mathcal{U})
$$

for some $\mu(0<\mu \leq 1)$, and let us denote $\mathcal{T}_{2,1} \equiv \mathcal{T}_{2}$. Furthermore, for some real $p$ with $0<p \leq 2$ we define a subclass $\mathcal{S}(p)$ of $\mathcal{A}$ consisting of all function

Received April 21, 2008.

2000 Mathematics Subject Classification. 30C45.

Key words and phrases. analytic functions, integral operator, univalent function.

Supported by the GAR 19/2008.

(C)2009 The Korean Mathematical Society 
$f(z)$ which satisfy

$$
\left|\left(\frac{z}{f(z)}\right)^{\prime \prime}\right| \leq p \quad(z \in \mathcal{U}) .
$$

In [9], Singh has shown that if $f(z) \in \mathcal{S}(p)$, then $f(z)$ satisfies

$$
\left|\frac{z^{2} f^{\prime}(z)}{(f(z))^{2}}-1\right| \leq p|z|^{2}, \quad(z \in \mathcal{U})
$$

L. V. Alfors in [1] and J. Becker in [2] has obtained the next univalence criterion:

Theorem 1.1. Let $c$ be a complex number, $|c| \leq 1, c \neq-1$. If $f(z)=$ $z+a_{2} z^{2}+\cdots$ is a regular function in $\mathcal{U}$ and

$$
\left.|c| z\right|^{2}+\left(1-|z|^{2}\right) \frac{z f^{\prime \prime}(z)}{f^{\prime}(z)} \mid \leq 1
$$

for all $z \in \mathcal{U}$, then the function $f$ is regular and univalent in $\mathcal{U}$.

In the paper [7], Pescar need the following theorem:

Theorem $1.2([7])$. Let $\beta$ be a complex number, $\boldsymbol{R e} \beta>0$, and $c$ a complex number, $|c| \leq 1, c \neq-1$ and $h(z)=z+a_{2} z^{2}+\cdots$, a regular function in $\mathcal{U}$. If

$$
\left.|c| z\right|^{2 \beta}+\left(1-|z|^{2 \beta}\right) \frac{z h^{\prime \prime}(z)}{\beta h^{\prime}(z)} \mid \leq 1
$$

for all the $z \in \mathcal{U}$, then the function

$$
F_{\beta}(z)=\left[\beta \int_{0}^{z} t^{\beta-1} h^{\prime}(t) d t\right]^{\frac{1}{\beta}}=z+\cdots
$$

is regular and univalent in $\mathcal{U}$.

The General Schwarz Lemma. Let the function $f(z)$ be regular in the disk $\mathcal{U}_{R}=\{z \in \mathbb{C} ;|z|<R\}$, with $|f(z)|<M$ for fixed $M$. If $f(z)$ has one zero with multiply $\geq m$ for $z=0$, then

$$
|f(z)| \leq \frac{M}{R^{m}}|z|^{m}, z \in \mathcal{U}_{R}
$$

The equality (in the inequality (1.5) for $z \neq 0$ ) can hold only if $f(z)=e^{i \theta} \frac{M}{R^{m}} z^{m}$, where $\theta$ is constant.

In the paper [8], Seenivasagan and Breaz consider for $f_{i} \in \mathcal{A}_{2}(i=1,2, \ldots, n)$ and $\alpha_{1}, \alpha_{2}, \ldots, \alpha_{n}, \beta \in \mathbb{C}$, the integral operator

$$
F_{\alpha_{1}, \alpha_{2}, \ldots, \alpha_{n}, \beta}(z)=\left\{\beta \int_{0}^{z} t^{\beta-1} \prod_{i=1}^{n}\left(\frac{f_{i}(t)}{t}\right)^{\frac{1}{\alpha_{i}}} d t\right\}^{\frac{1}{\beta}} .
$$

When $\alpha_{i}=\alpha$ for all $i=1,2, \ldots, n, F_{\alpha_{1}, \alpha_{2}, \ldots, \alpha_{n}, \beta}(z)$ becomes the integral operator $F_{\alpha, \beta}$ considered in [3]. 


\section{Main results}

Theorem 2.1. Let $M_{i} \geq 1$ for all $i \in\{1, \ldots, n\}$, c be a complex number and the functions $f_{i} \in \mathcal{S}\left(p_{i}\right)$ for $i \in\{1, \ldots, n\}$ satisfying the condition (1.4). Consider $\alpha_{i}, \beta$ be a complex number with the property $\operatorname{Re} \beta \geq \sum_{i=1}^{n} \frac{\left(1+p_{i}\right) M_{i}+1}{\left|\alpha_{i}\right|}$. If

$$
|c| \leq 1-\frac{1}{\operatorname{Re} \beta} \sum_{i=1}^{n} \frac{\left(1+p_{i}\right) M_{i}+1}{\left|\alpha_{i}\right|}
$$

and

$$
\left|f_{i}(z)\right| \leq M_{i}
$$

for all $z \in \mathcal{U}$ and $i \in\{1, \ldots, n\}$, then the function $F_{\alpha_{1}, \alpha_{2}, \ldots, \alpha_{n}, \beta}$ defined in (1.6) is univalent.

Proof. Define a function

$$
h(z)=\int_{0}^{z} \prod_{i=1}^{n}\left(\frac{f_{i}(t)}{t}\right)^{\frac{1}{\alpha_{i}}} d t
$$

then we have $h(0)=h^{\prime}(0)-1=0$. Also a simple computation yields

$$
\frac{z h^{\prime \prime}(z)}{h^{\prime}(z)}=\sum_{i=1}^{n} \frac{1}{\alpha_{i}}\left(\frac{z f_{i}^{\prime}(z)}{f_{i}(z)}-1\right) \text {. }
$$

From equality (2.2), we have

$$
\left|\frac{z h^{\prime \prime}(z)}{h^{\prime}(z)}\right| \leq \sum_{i=1}^{n} \frac{1}{\left|\alpha_{i}\right|}\left(\left|\frac{z f_{i}^{\prime}(z)}{f_{i}(z)}\right|+1\right)=\sum_{i=1}^{n} \frac{1}{\left|\alpha_{i}\right|}\left(\left|\frac{z^{2} f_{i}^{\prime}(z)}{f_{i}^{2}(z)}\right|\left|\frac{f_{i}(z)}{z}\right|+1\right) .
$$

From the hypothesis, we have $\left|f_{i}(z)\right| \leq M_{i}, z \in \mathcal{U}$ and $i \in\{1, \ldots, n\}$, then by General Schwarz Lemma, we obtain that

$$
\left|f_{i}(z)\right| \leq M_{i}|z|
$$

for all $z \in \mathcal{U}$ and $i \in\{1, \ldots, n\}$.

We apply this result in inequality (2.3), we obtain

$$
\begin{aligned}
\left|\frac{z h^{\prime \prime}(z)}{h^{\prime}(z)}\right| & \leq \sum_{i=1}^{n} \frac{1}{\left|\alpha_{i}\right|}\left(\left|\frac{z^{2} f_{i}^{\prime}(z)}{\left(f_{i}(z)\right)^{2}}\right| M_{i}+1\right) \\
& \leq \sum_{i=1}^{n} \frac{1}{\left|\alpha_{i}\right|}\left(\left|\frac{z^{2} f_{i}^{\prime}(z)}{\left(f_{i}(z)\right)^{2}}-1\right| M_{i}+M_{i}+1\right) \\
& =\sum_{i=1}^{n} \frac{1}{\left|\alpha_{i}\right|}\left(p_{i} M_{i}|z|^{2}+M_{i}+1\right)<\sum_{i=1}^{n} \frac{\left(1+p_{i}\right) M_{i}+1}{\left|\alpha_{i}\right|} .
\end{aligned}
$$


Next, we evaluate the expression:

$$
\begin{aligned}
& \left.|c| z\right|^{2 \beta}+\left(1-|z|^{2 \beta}\right) \frac{z h^{\prime \prime}(z)}{\beta h^{\prime}(z)} \mid \\
\leq & |c|+\frac{1}{|\beta|}\left|\frac{z h^{\prime \prime}(z)}{h^{\prime}(z)}\right| \leq|c|+\frac{1}{|\beta|} \sum_{i=1}^{n} \frac{\left(1+p_{i}\right) M_{i}+1}{\left|\alpha_{i}\right|} \\
< & |c|+\frac{1}{\operatorname{Re} \beta} \sum_{i=1}^{n} \frac{\left(1+p_{i}\right) M_{i}+1}{\left|\alpha_{i}\right|} .
\end{aligned}
$$

So, from (2.1) we have:

$$
\left.|c| z\right|^{2 \beta}+\left(1-|z|^{2 \beta}\right) \frac{z h^{\prime \prime}(z)}{\beta h^{\prime}(z)} \mid \leq 1 .
$$

Applying Theorem 1.2, we obtain that $F_{\alpha_{1}, \alpha_{2}, \ldots, \alpha_{n}, \beta}$ is univalent.

Corollary 2.2. Let $M \geq 1, c$ be a complex number and the functions $f_{i} \in \mathcal{S}(p)$ for $i \in\{1, \ldots, n\}$ satisfying the condition (1.4). Consider $\alpha_{i}, \beta$ be a complex numbers with the property $\operatorname{Re} \beta \geq \sum_{i=1}^{n} \frac{(1+p) M+1}{\left|\alpha_{i}\right|}$. If

$$
|c| \leq 1-\frac{1}{\operatorname{Re} \beta} \sum_{i=1}^{n} \frac{(1+p) M+1}{\left|\alpha_{i}\right|}
$$

and

$$
\left|f_{i}(z)\right| \leq M
$$

for all $z \in \mathcal{U}$, then the function $F_{\alpha_{1}, \alpha_{2}, \ldots, \alpha_{n}, \beta}$ defined in (1.6) is univalent.

Proof. We consider in Theorem $2.1 M_{1}=M_{2}=\cdots=M_{n}=M$.

Corollary 2.3. Let $M_{i} \geq 1$ for $i \in\{1, \ldots, n\}$, $c$ be a complex number and the functions $f_{i} \in \mathcal{S}\left(p_{i}\right)$, for $i \in\{1, \ldots, n\}$ satisfying the condition (1.4). Consider $\alpha, \beta$ be a complex numbers, $\boldsymbol{R e} \beta \geq \sum_{i=1}^{n} \frac{\left(\left(1+p_{i}\right) M_{i}+1\right)}{|\alpha|}$. If

$$
|c| \leq 1-\frac{1}{\operatorname{Re} \beta} \sum_{i=1}^{n} \frac{\left(\left(p_{i}+1\right) M_{i}+1\right)}{|\alpha|}
$$

and

$$
\left|f_{i}(z)\right| \leq M_{i}
$$

for all $z \in \mathcal{U}$ and $i \in\{1, \ldots, n\}$, then the function

$$
F_{\alpha, \beta}(z)=\left\{\beta \int_{0}^{z} t^{\beta-1} \prod_{i=1}^{n}\left(\frac{f_{i}(t)}{t}\right)^{\frac{1}{\alpha}} d t\right\}^{\frac{1}{\beta}}
$$

is univalent.

Proof. In Theorem 2.1 we consider $\alpha_{1}=\alpha_{2}=\cdots=\alpha_{n}=\alpha$. 
Corollary 2.4. Let $M \geq 1$, c be a complex number and the function $f \in$ $\mathcal{S}(p)$, satisfy the condition (1.4). Consider $\alpha, \beta$ be a complex numbers with the property $\boldsymbol{\operatorname { R e }} \beta \geq \frac{(1+p) M+1}{|\alpha|}$. If

$$
|c| \leq 1-\frac{(1+p) M+1}{\beta|\alpha|}
$$

and

$$
|f(z)| \leq M
$$

for all $z \in \mathcal{U}$, then the function

$$
G_{\alpha, \beta}(z)=\left\{\beta \int_{0}^{z} t^{\beta-1}\left(\frac{f(t)}{t}\right)^{\frac{1}{\alpha}} d t\right\}^{\frac{1}{\beta}}
$$

is univalent.

Proof. In Theorem 2.1 we consider $n=1$.

Theorem 2.5. Let $M_{i} \geq 1$ for all $i \in\{1, \ldots, n\}, c$ a complex number and the functions $f_{i} \in \mathcal{T}_{2, \mu_{i}}$ for $i \in\{1, \ldots, n\}$ satisfy the condition (1.3). We consider $\alpha_{i}, \beta$ be a complex numbers with the property $\operatorname{Re} \beta \geq \sum_{i=1}^{n} \frac{\left(1+\mu_{i}\right) M_{i}+1}{\left|\alpha_{i}\right|}$. If

$$
|c| \leq 1-\frac{1}{\operatorname{Re} \beta} \sum_{i=1}^{n} \frac{\left(1+\mu_{i}\right) M_{i}+1}{\left|\alpha_{i}\right|}
$$

and

$$
\left|f_{i}(z)\right| \leq M_{i}
$$

for all $z \in \mathcal{U}$ and $i \in\{1, \ldots, n\}$, then the function $F_{\alpha_{1}, \alpha_{2}, \ldots, \alpha_{n}, \beta}$ defined in (1.6) is univalent.

Proof. Define a function

$$
h(z)=\int_{0}^{z} \prod_{i=1}^{n}\left(\frac{f_{i}(t)}{t}\right)^{\frac{1}{\alpha_{i}}} d t
$$

then we have $h(0)=h^{\prime}(0)-1=0$. After the same steps with the Theorem 2.1 we have:

$$
\begin{aligned}
\left|\frac{z h^{\prime \prime}(z)}{h^{\prime}(z)}\right| & \leq \sum_{i=1}^{n} \frac{1}{\left|\alpha_{i}\right|}\left(\left|\frac{z^{2} f_{i}^{\prime}(z)}{\left(f_{i}(z)\right)^{2}}\right| M_{i}+1\right) \\
& \leq \sum_{i=1}^{n} \frac{1}{\left|\alpha_{i}\right|}\left(\left|\frac{z^{2} f_{i}^{\prime}(z)}{\left(f_{i}(z)\right)^{2}}-1\right| M_{i}+M_{i}+1\right) \\
& =\sum_{i=1}^{n} \frac{1}{\left|\alpha_{i}\right|}\left(\mu_{i} M_{i}+M_{i}+1\right)<\sum_{i=1}^{n} \frac{\left(1+\mu_{i}\right) M_{i}+1}{\left|\alpha_{i}\right|}
\end{aligned}
$$


We evaluate the next expression:

$$
\begin{aligned}
& \left.|c| z\right|^{2 \beta}+\left(1-|z|^{2 \beta}\right) \frac{z h^{\prime \prime}(z)}{\beta h^{\prime}(z)} \mid \\
\leq & |c|+\frac{1}{|\beta|}\left|\frac{z h^{\prime \prime}(z)}{h^{\prime}(z)}\right| \leq|c|+\frac{1}{|\beta|} \sum_{i=1}^{n} \frac{\left(1+\mu_{i}\right) M_{i}+1}{\left|\alpha_{i}\right|} \\
< & |c|+\frac{1}{\operatorname{Re} \beta} \sum_{i=1}^{n} \frac{\left(1+\mu_{i}\right) M_{i}+1}{\left|\alpha_{i}\right|} .
\end{aligned}
$$

So, from (2.4) we have:

$$
\left.|c| z\right|^{2 \beta}+\left(1-|z|^{2 \beta}\right) \frac{z h^{\prime \prime}(z)}{\beta h^{\prime}(z)} \mid \leq 1 .
$$

Applying Theorem 1.2, we obtain that $F_{\alpha_{1}, \alpha_{2}, \ldots, \alpha_{n}, \beta}$ is univalent.

Corollary 2.6. Let $M \geq 1$, c be a complex number and the functions $f_{i} \in \mathcal{T}_{2, \mu_{i}}$ for $i \in\{1, \ldots, n\}$ satisfy the condition (1.3). We consider $\alpha_{i}, \beta$ be a complex numbers with the property $\operatorname{Re} \beta \geq \sum_{i=1}^{n} \frac{\left(1+\mu_{i}\right) M+1}{\left|\alpha_{i}\right|}$. If

$$
|c| \leq 1-\frac{1}{\operatorname{Re} \beta} \sum_{i=1}^{n} \frac{\left(1+\mu_{i}\right) M+1}{\left|\alpha_{i}\right|}
$$

and

$$
\left|f_{i}(z)\right| \leq M
$$

for all $z \in \mathcal{U}$ and $i \in\{1, \ldots, n\}$, then the function $F_{\alpha_{1}, \alpha_{2}, \ldots, \alpha_{n}, \beta}$ defined in (1.6) is univalent.

Proof. We consider in Theorem 2.5 $M_{1}=M_{2}=\cdots=M_{n}=M$.

Corollary 2.7. Let $M_{i} \geq 1$ for $i \in\{1, \ldots, n\}$, c be a complex number and the functions $f_{i} \in \mathcal{T}_{2, \mu_{i}}$ for $i \in\{1, \ldots, n\}$ satisfy the condition (1.3). We consider $\alpha, \beta$ be a complex numbers with the property $\operatorname{Re} \beta \geq \sum_{i=1}^{n} \frac{\left(\left(1+\mu_{i}\right) M_{i}+1\right)}{|\alpha|}$. If

$$
|c| \leq 1-\frac{1}{\operatorname{Re} \beta} \sum_{i=1}^{n} \frac{\left(\left(1+\mu_{i}\right) M_{i}+1\right)}{|\alpha|}
$$

and

$$
\left|f_{i}(z)\right| \leq M_{i}
$$

for all $z \in \mathcal{U}$ and $i \in\{1, \ldots, n\}$, then the function

$$
F_{\alpha, \beta}(z)=\left\{\beta \int_{0}^{z} t^{\beta-1} \prod_{i=1}^{n}\left(\frac{f_{i}(t)}{t}\right)^{\frac{1}{\alpha}} d t\right\}^{\frac{1}{\beta}}
$$

is univalent.

Proof. In Theorem 2.5 we consider $\alpha_{1}=\alpha_{2}=\cdots=\alpha_{n}=\alpha$. 
Corollary 2.8. Let $M \geq 1$, $c$ be a complex number and the function $f \in \mathcal{T}_{2, \mu}$ satisfy the condition (1.3). We consider $\alpha, \beta$ be a complex numbers with the property $\operatorname{Re} \beta \geq \frac{(1+\mu) M+1}{|\alpha|}$. If

$$
|c| \leq 1-\frac{1}{\operatorname{Re} \beta \mid} \frac{(1+\mu) M+1}{|\alpha|}
$$

and

$$
|f(z)| \leq M
$$

for all $z \in \mathcal{U}$, then the function

$$
G_{\alpha, \beta}(z)=\left\{\beta \int_{0}^{z} t^{\beta-1}\left(\frac{f(t)}{t}\right)^{\frac{1}{\alpha}} d t\right\}^{\frac{1}{\beta}}
$$

is univalent.

Proof. In Theorem 2.5 we consider $n=1$.

Corollary 2.9. Let $M_{i} \geq 1$ for all $i \in\{1, \ldots, n\}, c$ a complex number and the functions $f_{i} \in \mathcal{T}$ for $i \in\{1, \ldots, n\}$ satisfy the condition (1.2). We consider $\alpha_{i}, \beta$ be a complex numbers with the property $\operatorname{Re} \beta \geq \sum_{i=1}^{n} \frac{2 M_{i}+1}{\left|\alpha_{i}\right|}$. If

$$
|c| \leq 1-\frac{1}{\operatorname{Re} \beta} \sum_{i=1}^{n} \frac{2 M_{i}+1}{\left|\alpha_{i}\right|}
$$

and

$$
\left|f_{i}(z)\right| \leq M_{i}
$$

for all $z \in \mathcal{U}$ and $i \in\{1, \ldots, n\}$, then the function $F_{\alpha_{1}, \alpha_{2}, \ldots, \alpha_{n}, \beta}$ defined in (1.6) is univalent.

Proof. After the same steps with the Theorem 2.5 we obtain the conclusion of this corollary.

\section{References}

[1] L. V. Ahlfors, Sufficient conditions for quasiconformal extension, Discontinuous groups and Riemann surfaces (Proc. Conf., Univ. Maryland, College Park, Md., 1973), pp. 23-29. Ann. of Math. Studies, No. 79, Princeton Univ. Press, Princeton, N.J., 1974.

[2] J. Becker, Löwnersche Differentialgleichung und Schlichtheitskriterien, Math. Ann. 202 (1973), 321-335.

[3] D. Breaz and N. Breaz, The univalent condition for an integral operator on the classes $S(\alpha)$ and $T_{2}$, Acta Univ. Apulensis Math. Inform. No. 9 (2005), 63-69.

[4] D. Breaz and H. O. Guney, On the univalence criterion of a general integral operator, J. Inequal. Appl. 2008 (2008), Art. ID 702715, 8 pp.

[5] Z. Nehari, Conformal Mapping, McGraw-Hill Book Co., Inc., New York, Toronto, London, 1952

[6] S. Ozaki and M. Nunokawa, The Schwarzian derivative and univalent functions, Proc. Amer. Math. Soc. 33 (1972), 392-394.

[7] V. Pescar, A new generalization of Ahlfors's and Becker's criterion of univalence, Bull. Malaysian Math. Soc. (2) 19 (1996), no. 2, 53-54. 
[8] N. Seenivasagan and D. Breaz, Certain sufficient conditions for univalence, Gen. Math. 15 (2007), no. 4, 7-15.

[9] V. Singh, On a class of univalent functions, Int. J. Math. Math. Sci. 23 (2000), no. 12, 855-857.

Department of Mathematics

"1 Decembrie 1918 " University

Alba Iulia, Romania

E-mail address: dbreaz@uab.ro 